\title{
Audiological profile of indoor cycling teachers
}

Centro Universitário de Várzea Grande UNIVAG, Várzea Grande, Mato Grosso, Brasil.

2 Centro Universitário de Várzea Grande - UNIVAG,Várzea Grande, Mato Grosso, Brasil; Hospital Universitário Júlio Muller da Universidade Federal do Mato Grosso - HUJM/UFMT, Cuiabá, Mato Grosso, Brasil.

Conflict of interests: Nonexistent

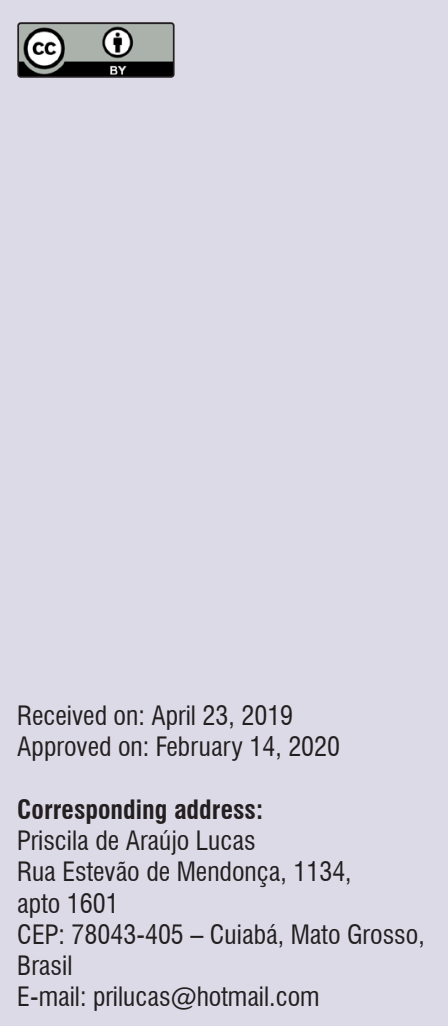

Kézzia Myrela da Costa ${ }^{1}$ https://orcid.org/0000-0002-1756-3577 Jorge Eto ${ }^{1}$ https://orcid.org/0000-0002-5492-1599 Priscila de Araújo Lucas ${ }^{2}$ https://orcid.org/0000-0002-8653-6441

\section{ABSTRACT}

Purpose: to describe the audiological profile and auditory complaints of indoor cycling teachers, as well as to relate the findings with time of noise exposure and presence of tinnitus.

Methods: participants were eight teachers of both genders, age ranging from 24 to 36 years, with professional experience in the area for at least one year. Sound pressure level was measured during the class, which lasted from 45 to 50 minutes and an adapted anamnesis questionnaire composed of ten items was applied to each individual to research the auditory symptoms and factors related to noise exposure and the audiological assessment performed. Inferential statistical tests were applied. The level of statistical significance was 0.05 .

Results: all of them had pure tone audiometry, tympanometry testing and brainstem auditory evoked potential within normal limits. There were alterations in the high frequency audiometry and otoacoustic emissions without a statistical correlation with the time of professional experience.

Conclusion: the audiological profile obtained was pure tone audiometry, tympanometry and brainstem auditory evoked potential within normal limits; altered transient otoacoustic and distortion product emissions and high frequency audiometry. The complaints reported were: tinnitus, dizziness, the need to listen at a high volume and being exposed to excessive noise.

Keywords: Physical Education and Training; Hearing;Occupational Noise 


\section{INTRODUCTION}

Indoor cycling (IC) is a gymnastics modality, practiced on a stationary bicycle, combining basic cycling movements with different musical rhythms, and a teacher guides the student so that he/she performs movements based on the musical stimulus ${ }^{1}$.

The indoor cycling emerged in the 1980s, as a new alternative for aerobic activity in gyms, through a continuous or interval training program, aimed at maintaining and improving the cardiovascular system ${ }^{2}$.

The reasons that lead men and women to practice indoor cycling are: pleasure in physical activity, esthetic, acquisition of better physical conditioning, quality of life. Moreover, the practice of indoor cycling is related to the promotion and maintenance of physical and psychological well-being by providing a moment of socialization and leisure ${ }^{3}$.

The IC class is taught by a teacher and lasts 45 to 50 minutes, the music is used to motivate students during the class ${ }^{4}$. The intensity of music varies between low, moderate and high based on the musical rhythm imposed by the teacher and the student's level of experience. However, as a way of motivating students, teachers have played music at a high volume, exceeding the acceptable and normative values, which is $80 \mathrm{~dB}^{5}$.

The strategy used as a tool for indoor cycling is the use of music and the impact it brings and the means by which music may serve as an adequate tool to manipulate the running cadence. It was shown that music resulted in better performance in indoor cycling activities, causing a positive and considerable effect ${ }^{6}$.

It was observed that music can influence the individuals' participation during physical activities, such as indoor cycling. For this reason, the strategy of using music during activities serves to encourage the exercise to be perceived more positively and may become more interesting for individuals. When music is listened during sports activities, it distracts fatigue, removes discomfort, improves mood, increases arousal, relieves stress, stimulates rhythmic movement and brings a feeling of increased energy to individuals ${ }^{3}$.

However, the IC modality is performed in environments that were not designed for this purpose, there is no type of acoustic treatment, thus impairing the auditory health of teachers 5 .The noise-induced hearing loss is the second most commonly found occupational disease, despite decades of studies, interventions in the workplace and regulations ${ }^{7}$.
The presence of continuous noise in a work environment can damage the workers' auditory system and cause hearing loss. Noise-induced hearing loss $(\mathrm{NIHL})$ is the alteration in hearing thresholds, of sensorineural type, resulting from systematic occupational exposure to high levels of sound pressure. Initially, the damage impairs hearing at the higher frequencies, around $4.000 \mathrm{~Hz}$, and then progressively affects the lower frequencies. Individuals only perceive this loss, which is irretrievable, when the frequencies of conversation are affected, which impairs their relationship with other persons. Moreover, if there is no decrease in noise exposure, hearing loss becomes worse ${ }^{8}$.

Studies have found that, besides the degenerative changes in outer and inner hair cells, there is the involvement of neuronal synapses proximal to the sensory cells and the degeneration of afferent nerve fibers of the cochlear nerve. These authors suggested two hypotheses that would explain neuronal damage: neural hyperactivity due to excessive acoustic stimulation and/or neuronal response to degeneration of hair cells ${ }^{9,10}$. The Brainstem Auditory Evoked Potential (BAEP) is a useful complementary test in the assessment of $\mathrm{NIHL}$ and demonstrates that besides the sensory damage, there is damage to the first neural afferent pathways of the auditory system ${ }^{11}$.

According to the World Health Organization (WHO), excessive exposure to noise can cause other health problems, the noise-induced hearing loss can be accompanied by a series of effects, such as: tinnitus, auditory stresses, increased adrenaline production, irritability, insomnia, recruitment (discomfort for loud sounds) alterations in speech perception ${ }^{12}$. Therefore, it is considered that the effects resulting from NIHL may be impairing the quality of life of IC teachers at work and social relationships ${ }^{13}$.

The purpose of this study was to describe the audiological profile and auditory complaints of indoor cycling teachers, as well as to relate the findings with time of noise exposure and presence of tinnitus.

\section{METHODS}

This study was conducted after approval by the Research Ethics Committee of the University Center of Várzea Grande, Mato Grosso, Brazil, under number number 2.112.529. The study participants signed the Informed Consent Form (ICF), where they were informed about the research content. 
It was a cross-sectional study, carried out in the years 2018 and 2019 in the institutions to be informed in case of approval of the article.

The study population was selected through a convenience sample, and ten teachers of both genders, age ranging between 24 and 36 years, with professional experience in the area for at least one year, were invited to participate in this study. However, only eight teachers participated in all study stages and composed the sample. After the teachers accepted the invitation, they signed the informed consent form. The study consisted of three stages: 1. Measurement of the Sound Pressure Level during indoor cycling classes. 2. Verification of auditory symptoms. 3. Audiological assessment.

Stage 1: To evaluate the sound pressure levels in the gyms, an application called "Decibelimeter version 3.2.4" was used, available on the Play Store of Android devices. The application served to measure the medium sound pressure level and the maximum and minimum sound levels during the period of a class that ranged from 45 to 50 minutes, being measured by the researcher herself/himself.

Stage 2: To assess the auditory symptoms, a questionnaire adapted from Campelo, L. M. P; (2007) (Annex 1) was applied.

Stage 3: The audiological assessment was composed by Meatoscopy, pure tone audiometry, high frequency audiometry, tympanometry testing, otoacoustic emissions and brainstem auditory evoked potential. The pure tone and high frequency audiometry was performed in an acoustic booth.

When performing meatoscopy, a Heine otoscope was used, the procedure was performed with the patient seated and was verified whether there was (or not) an impediment for audiological assessment. In case of any impediment, patients were referred to the otorhinolaryngologist, and after the intervention, the patient returned to a new meatoscopy and assessment. The study patients had 14 hours of auditory rest to perform the tests.

Hearing thresholds were investigated through pure tone audiometry that measures the hearing acuity threshold from 250 to $8000 \mathrm{~Hz}$. This test was performed using the Inventis audiometer and Piano Plus model. The supra-earphone used was TDH39 model by Telephonics.

For a complementary assessment, high frequency audiometry, which measures the hearing acuity threshold from 9000 to $16000 \mathrm{~Hz}$, was conducted. The assessment was carried out using the Inventis and
Piano Plus audiometer. The supra-headset used was the Sennheiser HDA300 model.

The test was carried out based on the international standards established in an appropriate environment for carrying it out and within an acoustic booth, so that the noise did not impair the record of hearing thresholds.

Transient Evoked Otoacoustic Emissions (TEOAE) and Distortion Product Otoacoustic Emissions (DPOAE) were performed. The stimulus used to evoke TEOAE was at $85 \mathrm{dBSPL}$ non-linear click. The stimulus used to capture DPOAE was the pure tone in a $2 \mathrm{~F} 1-\mathrm{F} 2$ ratio and frequencies from 2000 to $5000 \mathrm{~Hz}$ are evaluated. The equipment used was the otoacoustic emissions analyzer Interacoustics, OtoRead model. It should be emphasized that this equipment model is ideal for neonatal hearing screening programs; however, it has flexibility and allows the choice of protocols for the assessment and analysis of signal-to-noise ratios at each frequency assessed.

The reference standard for TEOAE is a level of minimum signal-to-noise ratio higher than or equal to $3 \mathrm{~dB}$, in the frequency ranges analyzed separately and are present in individuals with audibility up to 25-30 dBHL. DPOAEs are considered as present when recorded at least $6 \mathrm{~dB}$ above background noise and are present in individuals with audibility of $45-50 \mathrm{dBHL}$.

To perform the tympanometry testing, the calibrated Madsen tympanometer and Zodiac model 901 was used. The acoustic immittance measure consists of assessing the moment when the tympanic membrane is at rest, when the tympanic membrane is under pressure variation and performs the investigation of the acoustic reflex, which is an involuntary contraction of the middle ear muscles in response to a sound stimulus that is given. Normal ear volume was considered from 0.3 to $1.6 \mathrm{ml}$ at a pressure of -100 to $+100 \mathrm{daPa}$ as the normal standard for tympanometry.

For the test, the patient was sitting on a chair and an earphone and a probe were placed in the outer ear. Tympanometry and investigation for the contralateral stapedial reflex were carried out at frequencies $500 \mathrm{~Hz}$, $1000 \mathrm{~Hz}, 2000 \mathrm{~Hz}, 4000 \mathrm{~Hz}$.

The BAEP was carried out with click acoustic stimulus on the rarefied polarity and presentation speed of 27.1 clicks per second and recording window of $12 \mathrm{~ms}$ and bandpass filter of $100 \mathrm{~Hz}$ and $3000 \mathrm{~Hz}$. For analyzing the generated trace, a total of 1000 clicks were presented twice so that reproducibility between the traces could be observed. The initial intensity was 
$80 \mathrm{~dB}$, with decreases of $20 \mathrm{~dB}$ to the lowest level at which wave $V$ was found and will be defined as the electrophysiological threshold.

The equipment used was the MEB $9400 \mathrm{NIHON}$ KOHDEN. During the test preparation, the skin was cleaned to place the electrodes using an abrasive paste and a conductive gel was applied to the electrodes. The surface electrodes were placed as follows: positive electrode placed on top of the forehead, reference electrode on the mastoid of the tested ear, ground electrode on the frontal region, the Elegamodelo DR531 supra-auricular phone was placed on the patient's assembly. The patient was comfortable, lying down and instructed to remain still and with good muscle relaxation.

Data from patient assessments were tabulated and analyzed through descriptive and inferential statistics, in order to answer the study results. Initially, the descriptive analysis was conducted (mean, minimum, maximum and standard deviation) of the performance time, class time, average, minimum and maximum sound pressure level in indoor cycling classes and the audibility thresholds obtained in pure tone and high frequency audiometry. The percentage analysis of the results of TEOAE and DPOAE was also carried out. The Pearson correlation test was used to verify the association between teachers' experience time and result of tests that were altered. The variables that found non-totalitarian results were the presence of tinnitus and the need to listen at a high volume. Thus, the Mann-Whitney test was used to verify the association between these variables with the experience time and the result of the TEOAE, DPOAE and hearing thresholds. The relation is a value that ranges from -1 to 1 , but, in order to facilitate reading and/or understanding, the values were transformed into a percentage (only multiplied by 100). The level of statistical significance was 0.05 .

\section{RESULTS}

In this study, eight indoor cycling teachers were assessed, 2 (25\%) being females and 6 (75\%) males, aged from 24 to 36 years. The assessed teachers denied previous history of otorrhea, otorrhagia and otalgia, as well as a family history of congenital or progressive deafness. The entire sample also denied using personal protective equipment (PPE) for hearing, during the teaching of IC classes. Next, the characterization of the sample, composed of the auditory symptoms and factors related to noise exposure, will be demonstrated.

Table 1 shows the characterization of the sample consisting of time of noise exposure and teachers' experience time and sound pressure levels during IC classes.

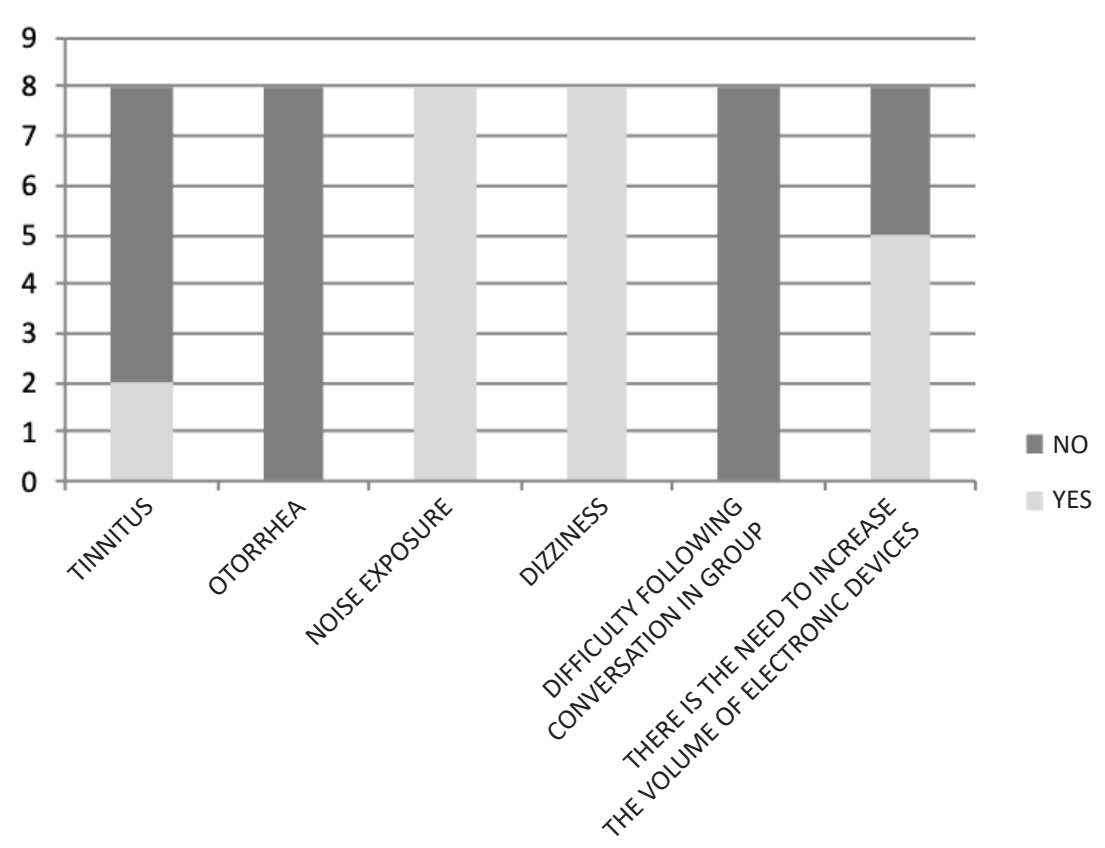

Figure 1. Characterization of auditory symptoms and factors related to noise exposure 
Table 1. Descriptive analysis of the time of noise exposure and teachers' performance and sound pressure levels of indoor cycling classes

\begin{tabular}{cccccc}
\hline & $\begin{array}{c}\text { Class time } \\
\text { (minutes) }\end{array}$ & $\begin{array}{c}\text { Minimum decibel } \\
\text { level }\end{array}$ & $\begin{array}{c}\text { Medium decibel } \\
\text { level }\end{array}$ & $\begin{array}{c}\text { Maximum decibel } \\
\text { level }\end{array}$ & $\begin{array}{c}\text { Experience time } \\
\text { (years) }\end{array}$ \\
\hline Mean & 35.8 & 91.5 & 106.1 & 117.2 & 6.7 \\
Minimum & 45 & 72 & 99 & 105 & 2 \\
Maximum & 50 & 110 & 115 & 121 & 14 \\
SD & 19.5 & 12.2 & 6.1 & 5.7 & 4.5 \\
\hline
\end{tabular}

Caption: SD: standard deviation

In the result of tympanometry testing, it was detected that the teachers did not have any alteration in the middle ear and in the research of contralateral acoustic reflexes, all participants obtained presence of reflexes during assessment. Regarding the assessment of brainstem auditory evoked potential, all showed absolute latencies and interpeak intervals and electrophysiological thresholds within normal limits, without showing signs of retrocochlear alteration.
In the result of pure tone audiometry, all individuals obtained hearing thresholds within normal range for all frequencies tested. Lowered hearing thresholds were observed in some individuals in high frequency audiometry, as shown in Figure 2.

Figure 3 displays the results of TEOAE, which shows greater absent responses in the frequency of $4000 \mathrm{~Hz}$.

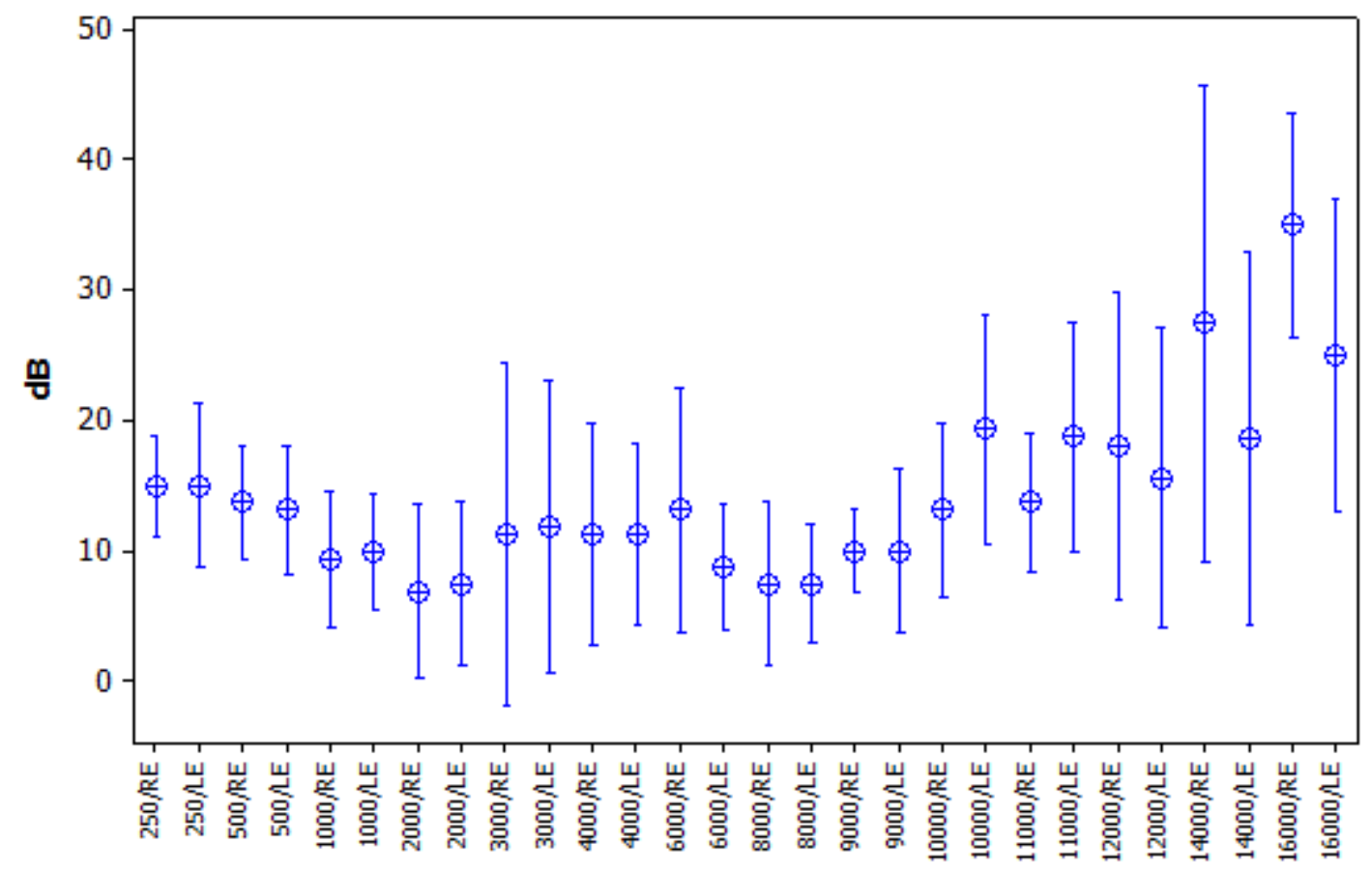

Caption: RE: right ear, LE: left ear

Figure 2. Characterization of hearing thresholds of the pure tone and high frequency audiometry 


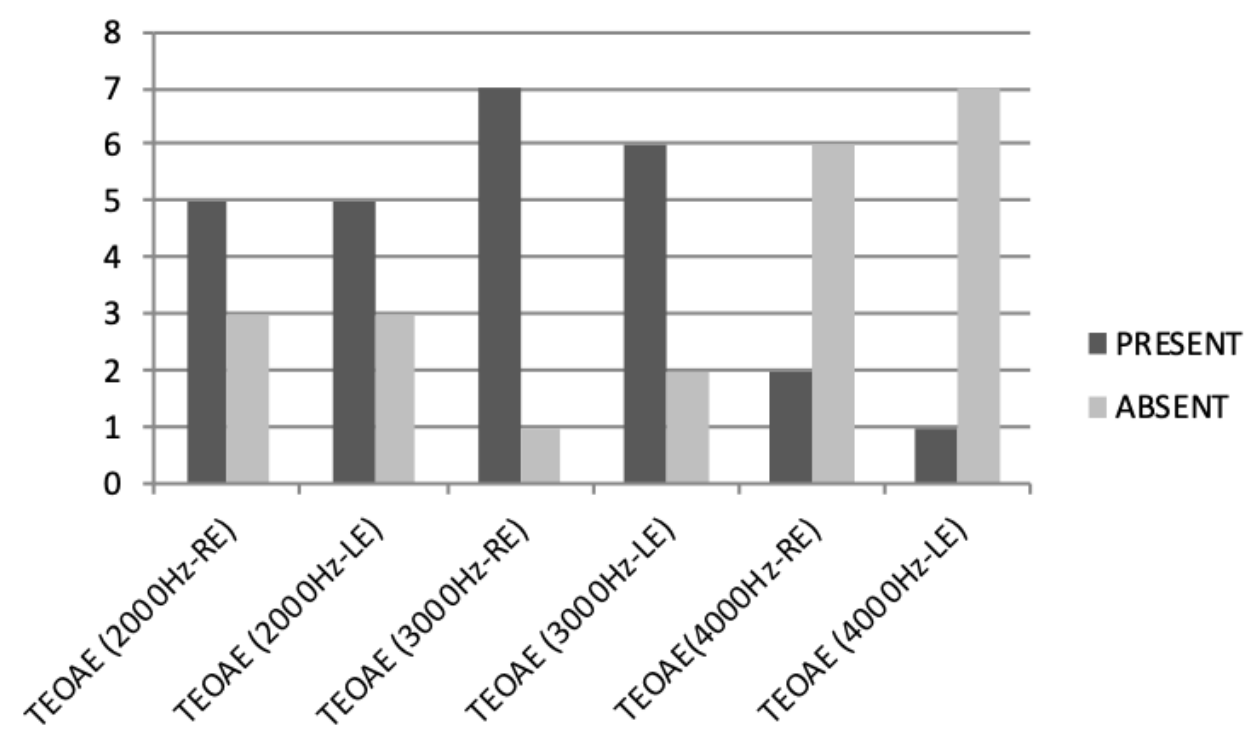

Legenda: OD: orelha direita, OE: orelha esquerda, Hz: Hertz, EOAT: Emissões Otoacústicas Evocadas Transientes

Figure 3. Characterization of the results of transient evoked otoacoustic emissions

Figure 4 shows the result of distortion product otoacoustic emissions, which did not change in most of the individuals assessed.
Table 2 refers to inferential statistical analysis to associate the experience time of IC teachers with the results of some tests applied. It is noticed that the Pearson Coefficient did not show an association between the variables.

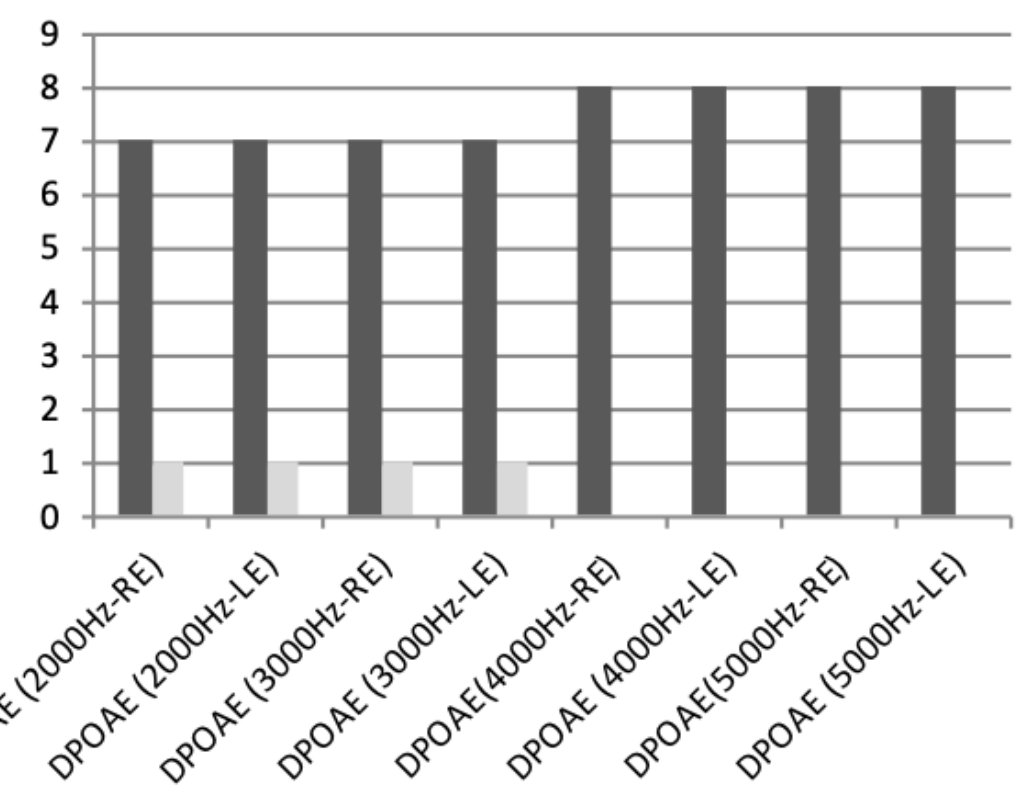

PRESENT

ABSENT

Caption: RE: right ear, LE: left ear, Hz: Hertz, DPOAE: Distortion Product Otoacoustic Emissions

Figure 4. Characterization of the result of distortion product evoked otoacoustic emissions 
Table 2. Association of the altered audiological tests with experience time

\begin{tabular}{ccc}
\hline & Corr $(\mathbf{r})$ & P-value \\
\hline TEOAE / 2000/RE & $0.0 \%$ & 1.000 \\
TEOAE / 2000/ LE & $-12.0 \%$ & 0.776 \\
TEOAE / 3000/ RE & $-18.5 \%$ & 0.661 \\
TEOAE / 3000/LE & $46.7 \%$ & 0.244 \\
TEOAE / 4000/RE & $-38.6 \%$ & 0.346 \\
TEOAE / 4000/LE & $8.6 \%$ & 0.839 \\
DPOAE / 2000/ RE & $-25.2 \%$ & 0.548 \\
DPOAE / 2000/ LE & $-43.1 \%$ & 0.286 \\
DPOAE / 3000/ RE & $-1.2 \%$ & 0.978 \\
DPOAE / 3000/ LE & $-22.9 \%$ & 0.586 \\
DPOAE / 4000/ RE & $28.9 \%$ & 0.487 \\
DPOAE / 4000/ LE & $3.6 \%$ & 0.932 \\
DPOAE /5000/RE & $55.4 \%$ & 0.154 \\
DPOAE /5000/ LE & $6.0 \%$ & 0.887 \\
9000/RE & $38.8 \%$ & 0.342 \\
9000/LE & $43.2 \%$ & 0.285 \\
10000/RE & $37.9 \%$ & 0.354 \\
10000/LE & $67.4 \%$ & 0.067 \\
11000/RE & $62.9 \%$ & 0.095 \\
11000/LE & $67.3 \%$ & 0.068 \\
12000/RE & $47.2 \%$ & 0.237 \\
12000/LE & $53.0 \%$ & 0.177 \\
14000/RE & $59.3 \%$ & 0.121 \\
14000/LE & $30.5 \%$ & 0.463 \\
16000/RE & $64.6 \%$ & 0.083 \\
16000/LE & $66.7 \%$ & 0.071 \\
\hline
\end{tabular}

*statistical test: Pearson correlation

Legenda: OD: orelha direita, OE: orelha esquerda, EOAT: Emissões Otoacústicas Transientes, EOAPD: Emissões Otoacústicas Produto de Distorção

Table 3 shows that there was statistical significance between the experience time and presence of tinnitus, where the average for "No" was 4.83 against 13.00 for those who answered "Yes" ( $p$-value $=0.044)$.
Table 4 shows that the need to listen at a high volume has a statistical significance in the results of: Transient OAE/ 3000/LE, 10000/RE, 11000/RE, 12000/ LE, 14000/LE and 16000/RE. 
Table 3. Comparison of the presence of tinnitus in relation to experience time, transient evoked otoacoustic emissions, distortion product evoked otoacoustic emissions and hearing thresholds

\begin{tabular}{|c|c|c|c|c|c|c|c|}
\hline Tinnitus & & Mean & Median & Standard Deviation & $\mathrm{N}$ & CI & P-value \\
\hline \multirow{2}{*}{ Experience time } & No & 4.83 & 4.5 & 2.99 & 6 & 2.40 & \multirow{2}{*}{0.044} \\
\hline & Yes & 13.00 & 13.0 & 1.41 & 2 & 1.96 & \\
\hline \multirow{2}{*}{ TEOAE 2000/RE } & No & 9.00 & 9.5 & 7.27 & 6 & 5.81 & \multirow{2}{*}{0.180} \\
\hline & Yes & 0.50 & 0.5 & 0.71 & 2 & 0.98 & \\
\hline \multirow{2}{*}{ TEOAE 2000/ LE } & No & 10.67 & 13.0 & 7.63 & 6 & 6.11 & \multirow{2}{*}{0.402} \\
\hline & Yes & 7.00 & 7.0 & 12.73 & 2 & 17.64 & \\
\hline \multirow{2}{*}{ TEOAE 3000/ RE } & No & 5.00 & 6.5 & 3.52 & 6 & 2.82 & \multirow{2}{*}{0.169} \\
\hline & Yes & 4.00 & 4.0 & 0.00 & 2 & $-x-$ & \\
\hline \multirow{2}{*}{ TEOAE 3000/LE } & No & 3.50 & 4.5 & 3.83 & 6 & 3.07 & \multirow{2}{*}{0.500} \\
\hline & Yes & 5.50 & 5.5 & 2.12 & 2 & 2.94 & \\
\hline \multirow{2}{*}{ TEOAE 4000/RE } & No & 1.83 & 1.0 & 5.08 & 6 & 4.06 & \multirow{2}{*}{0.502} \\
\hline & Yes & -1.00 & -1.0 & 0.00 & 2 & $-x-$ & \\
\hline \multirow{2}{*}{ TEOAE 4000/LE } & No & -1.00 & -1.0 & 2.28 & 6 & 1.82 & \multirow{2}{*}{0.864} \\
\hline & Yes & -1.00 & -1.0 & 5.66 & 2 & 7.84 & \\
\hline \multirow{2}{*}{ DPOAE / 2000/ RE } & No & 23.00 & 25.5 & 8.39 & 6 & 6.71 & \multirow{2}{*}{0.317} \\
\hline & Yes & 12.50 & 12.5 & 16.26 & 2 & 22.54 & \\
\hline \multirow{2}{*}{ DPOAE / 2000/ LE } & No & 19.67 & 19.0 & 9.83 & 6 & 7.87 & \multirow{2}{*}{0.505} \\
\hline & Yes & 13.00 & 13.0 & 11.31 & 2 & 15.68 & \\
\hline \multirow{2}{*}{ DPOAE / 3000/ RE } & No & 25.50 & 26.5 & 6.09 & 6 & 4.87 & \multirow{2}{*}{0.739} \\
\hline & Yes & 15.50 & 15.5 & 20.51 & 2 & 28.42 & \\
\hline \multirow{2}{*}{ DPOAE / 3000/ LE } & No & 26.83 & 28.0 & 8.42 & 6 & 6.74 & \multirow{2}{*}{0.402} \\
\hline & Yes & 14.00 & 14.0 & 21.21 & 2 & 29.40 & \\
\hline \multirow{2}{*}{ DPOAE / 4000/ RE } & No & 14.17 & 12.5 & 5.78 & 6 & 4.62 & 0.867 \\
\hline & Yes & 11.50 & 11.5 & 0.71 & 2 & 0.98 & \\
\hline DPOAE / 4000/ LE & No & 15.33 & 14.0 & 5.82 & 6 & 4.66 & 0.500 \\
\hline & Yes & 11.00 & 11.0 & 1.41 & 2 & 1.96 & 0.500 \\
\hline DPOAE / 5000/ RE & No & 10.33 & 9.5 & 4.13 & 6 & 3.31 & 0.241 \\
\hline & Yes & 14.00 & 14.0 & 4.24 & 2 & 5.88 & 0.641 \\
\hline DPOAE /5000/ LE & No & 13.50 & 13.0 & 6.12 & 6 & 4.90 & 0.867 \\
\hline & Yes & 12.00 & 12.0 & 2.83 & 2 & 3.92 & \\
\hline 250/RE & No & 15.00 & 15.0 & 4.47 & 6 & 3.58 & 1.000 \\
\hline $200 / n^{2}$ & Yes & 15.00 & 15.0 & 7.07 & 2 & 9.80 & 1.000 \\
\hline $250 / 1 \mathrm{~F}-2>$ & No & 15.83 & 15.0 & 8.61 & 6 & 6.89 & 0731 \\
\hline$\angle 00 / \mathrm{LE}$ & Yes & 12.50 & 12.5 & 3.54 & 2 & 4.90 & 0.101 \\
\hline 500/RE & No & 14.17 & 15.0 & 5.85 & 6 & 4.68 & 0604 \\
\hline & Yes & 12.50 & 12.5 & 3.54 & 2 & 4.90 & 0.004 \\
\hline $500 /$ LE & No & 13.33 & 12.5 & 6.83 & 6 & 5.47 & 1000 \\
\hline J00/LL & Yes & 12.50 & 12.5 & 3.54 & 2 & 4.90 & 1.000 \\
\hline 1000/RE & No & 9.17 & 10.0 & 3.76 & 6 & 3.01 & 1000 \\
\hline $1000 / 1 \mathrm{~L}$ & Yes & 10.00 & 10.0 & 14.14 & 2 & 19.60 & 1.000 \\
\hline 1000/LF & No & 8.33 & 7.5 & 4.08 & 6 & 3.27 & 0.161 \\
\hline 1000/LL & Yes & 15.00 & 15.0 & 7.07 & 2 & 9.80 & 0.101 \\
\hline $2000 / \mathrm{RE}$ & No & 4.17 & 5.0 & 3.76 & 6 & 3.01 & 0.211 \\
\hline $2000 / \mathrm{nL}$ & Yes & 15.00 & 15.0 & 14.14 & 2 & 19.60 & 0.211 \\
\hline
\end{tabular}




\begin{tabular}{|c|c|c|c|c|c|c|c|}
\hline Tinnitus & & Mean & Median & Standard Deviation & $\mathbf{N}$ & $\mathrm{Cl}$ & P-value \\
\hline \multirow{2}{*}{ 2000/LE } & No & 5.00 & 5.0 & 3.16 & 6 & 2.53 & \multirow{2}{*}{0.252} \\
\hline & Yes & 15.00 & 15.0 & 14.14 & 2 & 19.60 & \\
\hline \multirow{2}{*}{$3000 / R E$} & No & 7.50 & 5.0 & 8.80 & 6 & 7.04 & \multirow{2}{*}{0.594} \\
\hline & Yes & 22.50 & 22.5 & 31.82 & 2 & 44.10 & \\
\hline \multirow{2}{*}{ 3000/LE } & No & 8.33 & 7.5 & 8.16 & 6 & 6.53 & \multirow{2}{*}{0.399} \\
\hline & Yes & 22.50 & 22.5 & 24.75 & 2 & 34.30 & \\
\hline \multirow{2}{*}{ 4000/RE } & No & 10.00 & 10.0 & 7.07 & 6 & 5.66 & \multirow{2}{*}{0.866} \\
\hline & Yes & 15.00 & 15.0 & 21.21 & 2 & 29.40 & \\
\hline \multirow{2}{*}{ 4000/LE } & No & 9.17 & 7.5 & 7.36 & 6 & 5.89 & \multirow{2}{*}{0.238} \\
\hline & Yes & 17.50 & 17.5 & 10.61 & 2 & 14.70 & \\
\hline \multirow{2}{*}{$6000 / R E$} & No & 12.50 & 12.5 & 11.73 & 6 & 9.38 & \multirow{2}{*}{0.737} \\
\hline & Yes & 15.00 & 15.0 & 14.14 & 2 & 19.60 & \\
\hline \multirow{2}{*}{ 6000/LE } & No & 8.33 & 7.5 & 6.06 & 6 & 4.85 & \multirow{2}{*}{0.726} \\
\hline & Yes & 10.00 & 10.0 & 7.07 & 2 & 9.80 & \\
\hline \multirow{2}{*}{$8000 / R E$} & No & 8.33 & 7.5 & 8.16 & 6 & 6.53 & \multirow{2}{*}{0.606} \\
\hline & Yes & 5.00 & 5.0 & 7.07 & 2 & 9.80 & \\
\hline \multirow{2}{*}{ 8000/LE } & No & 6.67 & 7.5 & 6.06 & 6 & 4.85 & \multirow{2}{*}{0.475} \\
\hline & Yes & 10.00 & 10.0 & 0.00 & 2 & $-x-$ & \\
\hline \multirow{2}{*}{$9000 / \mathrm{RE}$} & No & 10.00 & 10.0 & 4.47 & 6 & 3.58 & \multirow{2}{*}{1.000} \\
\hline & Yes & 10.00 & 10.0 & 0.00 & 2 & $-x-$ & \\
\hline \multirow{2}{*}{ 9000/LE } & No & 10.83 & 10.0 & 8.61 & 6 & 6.89 & \multirow{2}{*}{0.731} \\
\hline & Yes & 7.50 & 7.5 & 3.54 & 2 & 4.90 & \\
\hline \multirow{2}{*}{ 10000/RE } & No & 10.83 & 12.5 & 4.92 & 6 & 3.93 & \multirow{2}{*}{0.387} \\
\hline & Yes & 20.00 & 20.0 & 14.14 & 2 & 19.60 & \\
\hline $1000011 \mathrm{~F}$ & No & 17.50 & 15.0 & 9.87 & 6 & 7.90 & 0475 \\
\hline 10U0U/LE & Yes & 25.00 & 25.0 & 14.14 & 2 & 19.60 & $0.4 / 5$ \\
\hline $11000 / \mathrm{BF}$ & No & 11.67 & 15.0 & 5.16 & 6 & 4.13 & 0124 \\
\hline 11000/nE & Yes & 20.00 & 20.0 & 7.07 & 2 & 9.80 & $0.1<4$ \\
\hline $11000 / 1 \mathrm{~F}$ & No & 17.50 & 15.0 & 12.14 & 6 & 9.72 & 0238 \\
\hline $11000 / \mathrm{LE}$ & Yes & 22.50 & 22.5 & 3.54 & 2 & 4.90 & 0.238 \\
\hline $12000 / \mathrm{RF}$ & No & 13.33 & 15.0 & 6.83 & 6 & 5.47 & 0200 \\
\hline I $2000 / \mathrm{KL}$ & Yes & 32.50 & 32.5 & 24.75 & 2 & 34.30 & 0.299 \\
\hline 12000/LF & No & 15.00 & 12.5 & 14.14 & 6 & 11.32 & 0867 \\
\hline & Yes & 17.50 & 17.5 & 17.68 & 2 & 24.50 & \\
\hline $14000 / \mathrm{RF}$ & No & 24.17 & 25.0 & 20.35 & 6 & 16.28 & 0606 \\
\hline 14000/KE & Yes & 37.50 & 37.5 & 31.82 & 2 & 44.10 & 0.000 \\
\hline $14000 / \mathrm{F}$ & No & 15.00 & 17.5 & 8.94 & 6 & 7.16 & 0399 \\
\hline 14000/LE & Yes & 32.50 & 32.5 & 24.75 & 2 & 34.30 & 0.399 \\
\hline $16000 / \mathrm{BF}$ & No & 33.33 & 32.5 & 9.83 & 6 & 7.87 & 0.604 \\
\hline IOUUO/KL & Yes & 40.00 & 40.0 & 14.14 & 2 & 19.60 & 0.004 \\
\hline & No & 20.00 & 17.5 & 11.40 & 6 & 9.12 & 0122 \\
\hline 16000/LE & Yes & 40.00 & 40.0 & 14.14 & 2 & 19.60 & 0.122 \\
\hline
\end{tabular}

* statistical test: Mann-Whitney

Caption: RE: right ear, LE: left ear, TEOAE: Transient Evoked Otoacoustic Emissions, DPOAE: Distortion Product Otoacoustic Emissions, N: sample number, Cl: confidence interva 
10/17 | Costa KM, Eto J, Lucas PA

Table 4. Comparison of the need to listen at a high volume to experience time, transient evoked otoacoustic emissions, distortion product otoacoustic emissions and hearing thresholds

\begin{tabular}{|c|c|c|c|c|c|c|c|}
\hline High Volume & & Mean & Median & Standard deviation & $\mathbf{N}$ & IC & P-value \\
\hline \multirow{2}{*}{ Experience time } & No & 6.33 & 5.0 & 5.13 & 3 & 5.81 & \multirow{2}{*}{0.764} \\
\hline & Yes & 7.20 & 6.0 & 4.82 & 5 & 4.22 & \\
\hline \multirow{2}{*}{ TEOAE 2000/RE } & No & 6.00 & 4.0 & 7.21 & 3 & 8.16 & \multirow{2}{*}{0.653} \\
\hline & Yes & 7.40 & 5.0 & 8.14 & 5 & 7.14 & \\
\hline \multirow{2}{*}{ TEOAE 2000/ LE } & No & 9.00 & 10.0 & 7.55 & 3 & 8.54 & \multirow{2}{*}{0.549} \\
\hline & Yes & 10.20 & 16.0 & 9.44 & 5 & 8.28 & \\
\hline \multirow{2}{*}{ TEOAE 3000/ RE } & No & 6.00 & 7.0 & 1.73 & 3 & 1.96 & \multirow{2}{*}{0.356} \\
\hline & Yes & 4.00 & 5.0 & 3.54 & 5 & 3.10 & \\
\hline \multirow{2}{*}{ TEOAE 3000/LE } & No & 0.67 & -1.0 & 2.89 & 3 & 3.27 & \multirow{2}{*}{0.050} \\
\hline & Yes & 6.00 & 6.0 & 1.87 & 5 & 1.64 & \\
\hline \multirow{2}{*}{ TEOAE 4000/RE } & No & 3.67 & 2.0 & 5.69 & 3 & 6.43 & \multirow{2}{*}{0.230} \\
\hline & Yes & -0.40 & -1.0 & 3.36 & 5 & 2.95 & \\
\hline \multirow{2}{*}{ TEOAE 4000/LE } & No & -2.33 & -1.0 & 2.31 & 3 & 2.61 & \multirow{2}{*}{0.219} \\
\hline & Yes & -0.20 & 0.0 & 3.11 & 5 & 2.73 & \\
\hline \multirow{2}{*}{ DPOAE / 2000/ RE } & No & 16.67 & 14.0 & 6.43 & 3 & 7.28 & \multirow{2}{*}{0.297} \\
\hline & Yes & 22.60 & 29.0 & 12.58 & 5 & 11.03 & \\
\hline \multirow{2}{*}{ DPOAE / 2000/ LE } & No & 23.00 & 21.0 & 12.12 & 3 & 13.72 & \multirow{2}{*}{0.297} \\
\hline & Yes & 15.00 & 18.0 & 8.12 & 5 & 7.12 & \\
\hline \multirow{2}{*}{ DPOAE / 3000/ RE } & No & 22.33 & 19.0 & 6.66 & 3 & 7.53 & \\
\hline & Yes & 23.40 & 27.0 & 12.90 & 5 & 11.30 & 0.655 \\
\hline & No & 28.00 & 29.0 & 9.54 & 3 & 10.79 & 0.368 \\
\hline DPUAE / 3000/ LE & Yes & 21.00 & 27.0 & 13.95 & 5 & 12.22 & 0.368 \\
\hline & No & 11.00 & 11.0 & 1.00 & 3 & 1.13 & 0368 \\
\hline DPUAE / $4000 /$ RE & Yes & 15.00 & 14.0 & 6.04 & 5 & 5.30 & 0.368 \\
\hline & No & 13.67 & 10.0 & 7.23 & 3 & 8.19 & \\
\hline DPUAE / 4000/ LE & Yes & 14.60 & 12.0 & 4.83 & 5 & 4.23 & 0.365 \\
\hline & No & 9.00 & 10.0 & 2.65 & 3 & 2.99 & \\
\hline DPUAE / 5000/ RE & Yes & 12.60 & 11.0 & 4.62 & 5 & 4.05 & 0.368 \\
\hline & No & 12.67 & 10.0 & 6.43 & 3 & 7.28 & 0653 \\
\hline DPUAE / $5000 /$ LE & Yes & 13.40 & 14.0 & 5.37 & 5 & 4.70 & 0.653 \\
\hline & No & 15.00 & 15.0 & 5.00 & 3 & 5.66 & \\
\hline 250/RE & Yes & 15.00 & 15.0 & 5.00 & 5 & 4.38 & 1.000 \\
\hline & No & 15.00 & 10.0 & 8.66 & 3 & 9.80 & \\
\hline 250/LE & Yes & 15.00 & 15.0 & 7.91 & 5 & 6.93 & 1.000 \\
\hline $500 / \mathrm{BF}$ & No & 11.67 & 10.0 & 7.64 & 3 & 8.64 & 0.439 \\
\hline SUO/RE & Yes & 15.00 & 15.0 & 3.54 & 5 & 3.10 & 0.439 \\
\hline $500 / \mathrm{F}$ & No & 10.00 & 10.0 & 5.00 & 3 & 5.66 & 0273 \\
\hline $500 / L E$ & Yes & 15.00 & 15.0 & 6.12 & 5 & 5.37 & 0.273 \\
\hline $1000 / \mathrm{BF}$ & No & 6.67 & 5.0 & 7.64 & 3 & 8.64 & \\
\hline 1000/RE & Yes & 11.00 & 10.0 & 5.48 & 5 & 4.80 & 0.356 \\
\hline $1000 / \mathrm{LF}$ & No & 8.33 & 10.0 & 2.89 & 3 & 3.27 & 0.638 \\
\hline 1000/LE & Yes & 11.00 & 10.0 & 6.52 & 5 & 5.71 & 0.030 \\
\hline 2000/RE & No & 3.33 & 5.0 & 2.89 & 3 & 3.27 & 0.337 \\
\hline & Yes & 9.00 & 5.0 & 9.62 & 5 & 8.43 & \\
\hline 2000/LE & No & 5.00 & 5.0 & 5.00 & 3 & 5.66 & 0608 \\
\hline$\angle O U O / L E$ & Yes & 9.00 & 5.0 & 8.94 & 5 & 7.84 & 0.608 \\
\hline & No & 6.67 & 0.0 & 11.55 & 3 & 13.07 & 0634 \\
\hline 3000/RE & Yes & 14.00 & 10.0 & 18.51 & 5 & 16.22 & 0.634 \\
\hline $3000 / \mathrm{LF}$ & No & 8.33 & 5.0 & 10.41 & 3 & 11.78 & 0.651 \\
\hline $3000 / \mathrm{LE}$ & Yes & 14.00 & 10.0 & 15.57 & 5 & 13.65 & 0.651 \\
\hline & No & 6.67 & 0.0 & 11.55 & 3 & 13.07 & \\
\hline 4000/RE & Yes & 14.00 & 10.0 & 9.62 & 5 & 8.43 & 0.291 \\
\hline
\end{tabular}




\begin{tabular}{|c|c|c|c|c|c|c|c|}
\hline High Volume & & Mean & Median & Standard deviation & $\mathbf{N}$ & IC & P-value \\
\hline \multirow{2}{*}{ 4000/LE } & No & 10.00 & 10.0 & 10.00 & 3 & 11.32 & \multirow{2}{*}{0.763} \\
\hline & Yes & 12.00 & 10.0 & 8.37 & 5 & 7.33 & \\
\hline \multirow{2}{*}{$6000 / \mathrm{RE}$} & No & 11.67 & 5.0 & 16.07 & 3 & 18.19 & \multirow{2}{*}{0.764} \\
\hline & Yes & 14.00 & 15.0 & 9.62 & 5 & 8.43 & \\
\hline \multirow{2}{*}{$6000 / \mathrm{LE}$} & No & 10.00 & 15.0 & 8.66 & 3 & 9.80 & \multirow{2}{*}{0.638} \\
\hline & Yes & 8.00 & 5.0 & 4.47 & 5 & 3.92 & \\
\hline \multirow{2}{*}{ 8000/RE } & No & 6.67 & 0.0 & 11.55 & 3 & 13.07 & \multirow{2}{*}{0.645} \\
\hline & Yes & 8.00 & 10.0 & 5.70 & 5 & 5.00 & \\
\hline \multirow{2}{*}{$8000 / \mathrm{LE}$} & No & 8.33 & 10.0 & 7.64 & 3 & 8.64 & \multirow{2}{*}{0.631} \\
\hline & Yes & 7.00 & 10.0 & 4.47 & 5 & 3.92 & \\
\hline \multirow{2}{*}{ 9000/RE } & No & 8.33 & 10.0 & 2.89 & 3 & 3.27 & \multirow{2}{*}{0.334} \\
\hline & Yes & 11.00 & 10.0 & 4.18 & 5 & 3.67 & \\
\hline \multirow{2}{*}{ 9000/LE } & No & 8.33 & 5.0 & 10.41 & 3 & 11.78 & \multirow{2}{*}{0.539} \\
\hline & Yes & 11.00 & 10.0 & 6.52 & 5 & 5.71 & \\
\hline \multirow{2}{*}{ 10000/RE } & No & 6.67 & 5.0 & 2.89 & 3 & 3.27 & \multirow{2}{*}{0.030} \\
\hline & Yes & 17.00 & 15.0 & 7.58 & 5 & 6.65 & \\
\hline \multirow{2}{*}{ 10000/LE } & No & 13.33 & 15.0 & 7.64 & 3 & 8.64 & \multirow{2}{*}{0.337} \\
\hline & Yes & 23.00 & 15.0 & 10.95 & 5 & 9.60 & \\
\hline \multirow{2}{*}{ 11000/RE } & No & 8.33 & 5.0 & 5.77 & 3 & 6.53 & \multirow{2}{*}{0.058} \\
\hline & Yes & 17.00 & 15.0 & 4.47 & 5 & 3.92 & \\
\hline \multirow[b]{2}{*}{ 11000/LE } & No & 13.33 & 15.0 & 7.64 & 3 & 8.64 & \multirow[b]{2}{*}{0.291} \\
\hline & Yes & 22.00 & 20.0 & 11.51 & 5 & 10.09 & \\
\hline \multirow[b]{2}{*}{ 12000/RE } & No & 11.67 & 15.0 & 5.77 & 3 & 6.53 & \multirow{2}{*}{0.216} \\
\hline & Yes & 22.00 & 20.0 & 16.81 & 5 & 14.73 & \\
\hline \multirow{2}{*}{ 12000/LE } & No & 3.33 & 5.0 & 2.89 & 3 & 3.27 & \multirow{2}{*}{0.024} \\
\hline & Yes & 23.00 & 20.0 & 12.04 & 5 & 10.55 & \\
\hline \multirow{2}{*}{ 14000/RE } & No & 13.33 & 15.0 & 12.58 & 3 & 14.24 & \multirow{2}{*}{0.167} \\
\hline & Yes & 36.00 & 25.0 & 22.75 & 5 & 19.94 & \\
\hline $110000 / 15$ & No & 8.33 & 10.0 & 7.64 & 3 & 8.64 & \\
\hline $14000 / \mathrm{LE}$ & Yes & 26.00 & 20.0 & 13.87 & 5 & 12.16 & 0.035 \\
\hline $16000 / \mathrm{RE}$ & No & 26.67 & 30.0 & 5.77 & 3 & 6.53 & $0 \cap 014$ \\
\hline $10000 / \mathrm{nL}$ & Yes & 40.00 & 35.0 & 9.35 & 5 & 8.20 & 0.044 \\
\hline $16000 / 4 F$ & No & 16.67 & 10.0 & 11.55 & 3 & 13.07 & 0219 \\
\hline & Yes & 30.00 & 30.0 & 14.58 & 5 & 12.78 & 0.219 \\
\hline
\end{tabular}

*statistical test: Mann-Whitney

Caption: RE: right ear, LE: left ear, TEOAE: Transient Evoked Otoacoustic Emissions, DPOAE: Distortion Product Otoacoustic Emissions, N: sample number, Cl: confidence interva

\section{DISCUSSION}

The present study shows that during the anamnesis and assessment questionnaire, the teachers, in their totality, reported that they feelt dizzy and were exposed to noise. All of them denied otorrhea and difficulties to follow conversation in groups. Some reported tinnitus and also the need of listening at a very loud volume, as shown in Figure1.

Studies show that age and time of exposure to high levels of sound pressure are important variables that favor the appearance of auditory disorders, and that the occurrence of hearing loss is related to factors linked to the individual characteristics of the person exposed to the sound pressure level, the environment and the aggressive agent itself (sound), thus, showing that teachers who work for a longer time are those who report more auditory symptoms ${ }^{1}$.

According to the literature, noise can disturb work, rest, sleep and communication in humans. Thus, when a person is exposed to loud noise levels, the reaction of the whole organism to this stimulus takes place, which is given by neurovegetative responses, that can become permanent, rising organic and psychological disorders $^{14,15}$.

Normally, individuals rarely exposed to high levels of sound pressure, can have hearing recovery after some time of auditory rest, since when the exposure becomes 
frequent, a permanent change in the threshold that is $\mathrm{NIHL}$ is gradually established ${ }^{16,17}$.

Chronic exposure to noise, even at very low levels, has the potential to cause a chronic increase in the stress hormone in humans and, thus, accelerate the aging of the myocardium and vascular walls. These effects are related to an increased risk of heart attack and other health effects, that is, immunosuppression ${ }^{14}$.

Table 1 shows the descriptive statistical analysis of the values of sound pressure levels in cycling classes at the gyms evaluated. It is noticed that the professionals work at a sound pressure level higher than that which can be supported by hearing based on NR15. The individual is exposed to the risk of permanent hearing loss when the time of daily exposure in the 8-hour working day exceeds the proposed noise of $85 \mathrm{~dB}$, according to the NR-15 of the Ordinance 3.214/78 of the Ministry of Labor ${ }^{15}$.

Considering the fact that the professional can work in more than one class per day, which in each class he/she is exposed around 45-50 minutes to high sound pressure values, and for a value of 85 $\mathrm{dB}$, the maximum time of daily exposure should be two hours (NR-15, 1994), it is possible to assume that the physical education teacher is working in an unhealthy environment. In an investigation carried out with teachers, it was shown that a large part of them worked at levels higher than $85 \mathrm{~dB}$, considered above the tolerance limits established by the legislation in force. Significant reductions in hearing sensitivity were found in the exposed subjects. This research reinforces that the auditory effects depend on the intensity and duration of noise exposure ${ }^{15}$.

A study showed that NIHL cases have grown as far as working hours have increased, and that workers with more than 20 years of business, that is, exposure, are the most affected ${ }^{17}$. This reinforces the statement that the auditory effects depend mainly on the intensity and duration of noise exposure, as shown ${ }^{18}$.

In this study, the results obtained in the pure tone audiometry showed that all participants were within normal limits. However, in the high frequency audiometry, a decline in the thresholds of some frequencies was observed for some individuals, as shown in Figure 2. There was a lowering of the hearing threshold in two teachers for both ears and the compromised frequencies varied: in one individual $(10,12,14$ and $16 \mathrm{KHz}$ bilaterally), and in another $(10,11,12 \mathrm{KHz}$ in the left ear and 14 and $16 \mathrm{KHz}$ in the right ear).
Research reports that the most affected frequency in $\mathrm{NIHL}$ cases is $4 \mathrm{KHz}$, with an initial impairment of 4 and $6 \mathrm{kHz}$ in the results obtained in conventional audiometries (pure tone audiometry) ${ }^{7}$.

When analyzing the average obtained at frequencies from 3000 to $8000 \mathrm{~Hz}$ in one study, it was observed that averages higher than $25 \mathrm{~dB}$ are at $6000 \mathrm{~Hz}$, that is, the hearing damage seems to affect mainly these frequencies ${ }^{12}$. Assessments carried out using pure tone audiometry indicate that $6000 \mathrm{~Hz}$ has been the first frequency to be affected, due to exposure to occupational noise ${ }^{12}$.

It is already a discussion topic of great studies in the field of hearing health that, in many cases, pure tone audiometry may not reveal a hearing loss in the beginning, because the NIHL initially affects the cochlea base, thus, compromising the high frequencies. TEOAEs are more sensitive to temporary changes in the audibility threshold than DPOAE and, the DPOAE are more effective in detecting changes in high frequencies ${ }^{15}$.

The pure tone audiometry does not consistently assess the response capacity of the cochlea base, a frequent site of changes acquired by noise exposure. However, the most appropriate assessment of this cochlear follow-up is through assessing the hearing thresholds of high frequencies ${ }^{19}$. The primary application of high frequency audiometry occurs in monitoring the hearing of individuals at risk of developing auditory disorders caused by exogenous or endogenous factors. It should be noted that there is still no consensus regarding the calibration standards for high frequencies. Studies that used high frequency audiometry to assess hearing in individuals exposed to occupational noise show that there is a worsening of hearing thresholds in both ears and that the thresholds in this group are worse than those peers do not exposed to occupational noise. These results indicate that high-frequency audiometry can provide evidence of injury by noise compared to pure tone audiometry, being the most sensitive to detect auditory changes due to noise exposure ${ }^{20,21}$.

Figure 3 shows the result obtained in the transient otoacoustic emissions of the individuals evaluated which the frequency $4000 \mathrm{~Hz}$ was the most affected. It is known that TEOAEs are more sensitive to detect cochlear changes ${ }^{22,23}$.

In a study carried out with 25 individuals assessed by recording evoked otoacoustic emissions and pure tone audiometry, pre and post-exposure to $100 \mathrm{~dB}$ 
$\mathrm{HL}$ for 10 minutes, there was alteration in TEOAE that allows detecting temporary changes in the hearing threshold. In this study, regarding the findings obtained with the TEOAE analysis, there was a greater occurrence of worsening in the mean amplitude of poststimulation response for high frequencies. The study also revealed that TEOAEs are more sensitive to noise exposure ${ }^{24}$.

A study carried out in military personnel using transient evoked otoacoustic emissions revealed that TEOAE is a clinical instrument that allows the detection of subtle cochlear alterations, before being revealed by pure tone audiometry. The authors found changes in the TEOAE amplitudes between 2000 and $4000 \mathrm{~Hz}$ and no change in the frequencies of 250 and $500 \mathrm{~Hz}^{23}$.

In comparison between pure tone audiometry and TEOAE, it was found that both showed sensitivity in detecting temporary changes in audibility thresholds and reproducibility after exposure to high levels of sound pressure, with pure tone audiometry being more efficient for frequencies from 3 to $8 \mathrm{kHz}$ and TEOAE for frequencies from 1 to $4 \mathrm{kHz}^{15}$.

When there is no TEOAE response, it suggests an initial lesion of the outer hair cells, so the symptom is often imperceptible, since it already shows an alteration in the basilar membrane and this can be identified early through the TEOAE ${ }^{25}$.

Studies report that there is a higher incidence of absent TEOAE among workers exposed to noise with pure tone audiometry within the normal range, showing that this is an effective test for the early detection of cochlear hearing loss ${ }^{26}$.

In Figure 4 was verified the DPOAE results of this research, there is an absence in the frequencies 2 $\mathrm{kHz}$ and $3 \mathrm{kHz}$ in one teacher only. The DPOAE is an important instrument for clinical assessment and monitoring of individuals exposed to high levels of sound pressure, because it analyzes the presence of frequency responses ${ }^{26}$. Research suggests that higher doses of exposure to occupational noise can cause greater proportions of cochlear lesions detectable by the DPOAE records. These results reinforce the idea that the DPOAE test can be useful in the identification of initial hearing disorders caused by noise, not yet detected by the pure tone audiometry test, as suggested by other studies 27,28 .

These studies mentioned above do not agree with the findings of this study, since the majority of the sample had present DPOAE responses, as shown in Figure 4. However, it is important to note that the only individual who had absent DPOAE was the one who had the longest experience time in indoor cycling.

Table 2 shows the association between experience time and results of TEOAE, DPOAE and high frequency audiometry. Pearson's coefficient must be above $82 \%$ to be considered as a significant association between variables. It is noticed, therefore, that there was no association between the experience time and TEOAE, DPOAE and hearing threshold, as well as there was no statistically significant relation between the experience time and the results of altered exams. Other studies show the direct relation between experience time of exposure to occupational noise and permanent hearing loss, which does not agree with the findings of this study ${ }^{15,25,28}$. It can be hypothesized that this relationship was not observed due to the small number of participants and heterogeneous experience time among the participants in this study.

The presence of tinnitus is strongly related in the literature to high levels of sound pressure and to the death of hair cells in the inner ear ${ }^{27-29}$. This sign seems to be an early indicator of hearing overload ${ }^{30-32}$. The exposure time to occupational noise influenced the presence of tinnitus in the sample of this study, which corroborates with a national study ${ }^{33}$, as shown in Table 3. At this point it is worth mentioning that the etiological multiplicity (metabolic, dental, pharmacological, cardiovascular, psychological, neurological disorders, among others) and the possibility of interaction between the various causes hinder the task of determining the cause of tinnitus. The individual may have a disease that causes tinnitus without having this causal relationship determined. Moreover, an etiological factor may not be enough to trigger tinnitus, but several factors acting synergistically.

Another symptom showed by most teachers was the need to listen to television or music at high intensity, in the presence of other people, to understand oral speech. This characteristic was associated with worse hearing thresholds at high frequencies, which is also described in the literature as indicative of stress or auditory fatigue with cochlear injury in basal regions, as shown in Table 4. Studies demonstrate ${ }^{34,35}$ that professionals exposed to unhealthy occupational noise have auditory processing difficulties with altered auditory abilities, which agrees with this study, since the need for louder intensity to understand speech in patients with normal hearing can denote alterations in central auditory abilities. It is noteworthy, however, that confirmation of alterations in central auditory abilities could 
only be carried out after auditory processing behavioral evaluation.

Finally, it is important to note that the brainstem auditory evoked potential in this study did not show any changes in any of the variables analyzed. However, studies with samples of individuals with $\mathrm{NIHL}$ report different results in relation to BAEP. The literature shows both absence of neural changes ${ }^{33-35}$ as well as evidence of impairments in the neural conduction of patients exposed to excessive noise ${ }^{36,37}$.

The literature establishes the positive influence that the cardiovascular system health has on maintaining the functions of the inner ear, particularly when exposed to excessive noise levels ${ }^{38}$. An international study shows that there seems to be a greater cochlear resistance to the harmful effects of noise in individuals with good blood oxygenation. Adept individuals to physical exercises and with integrity of the cardiovascular system show rapid recovery from the temporary change in hearing threshold after noise exposure ${ }^{39}$. It is noteworthy, however, that these studies do not exclude the potential damage to hearing, due to excessive noise.

The literature defends the benefits of earplugs for hearing health ${ }^{40}$. Thinking about keeping music as a motivational factor during IC classes, it can be suggested that earplugs are worn to perform activity in order to avoid impairing auditory functions due to excessive sound pressure levels.

Thus, it is noted that the limitation of this research refers to the small number of participants involved associated with the heterogeneity of time of noise exposure in the sample. The sample was recruited for convenience, and the ideal sample calculation was not performed. It is suggested, therefore, that further studies increase the sample to make it more homogeneous and robust and that the results are disseminated to physical education teachers, aiming at better conditions and greater awareness about hearing health.

\section{CONCLUSION}

The audiological profile of indoor cycling teachers obtained was within normal limits for pure tone audiometry, tympanometry and brainstem auditory evoked potential. Transient Evoked Otoacoustic and Distortion Product Otoacoustic Emissions and high frequency audiometry were altered. The main reports during the assessment of indoor cycling teachers were: tinnitus, dizziness, the need to listen at a high volume and being exposed to excessive noise.

\section{ACKNOWLEDGEMENTS}

To Prof. Dr. Lúcia Helena Gaeta Aleixo, dean of postgraduate, research and extension at UNIVAG and Prof. Ms. Flávio H.S. Foguel, vice-dean at UNIVAG for the incentive offered to professors to conduct this scientific research at the educational institution.

\section{REFERENCES}

1. Martins MVS. Influência de diferentes níveis de pressão sonora no limiar auditivo de professores de ciclismo indoor [dissertação]. Curitiba (PR): Universidade Federal do Paraná; 2015.

2. Vilarinho R, Garcez S, Rodrigues WYTC, Ahlin JV, Guedes JDP, Madureira BF. Efeitos do ciclismo indoor na composição corporal, resistência muscular, flexibilidade, equilíbrio e atividades cotidianas em idosos fisicamente ativos. Fit Perf J. 2009;8(6):446-51.

3. Moura NLG, Merida DEM, Campanelli JRMF. A influência motivacional da música em mulheres praticantes de ginástica de academia praticantes de ginástica de academia. REMEFE. 2007;6(3):103-18.

4. Milano F, Palma A, Assis M. Saúde e trabalho dos professores de educação física que atuam com ciclismo indoor. LectEducFis Deportes. 2007;(12):109-14.

5. Marcon CR, Zannnin PHT. Avaliação do ruído gerado por academias de ginástica. REEC. 2004;1(9):39-42.

6. Van DE, Moens B, Buhmann J, Demey M, Coorevits E, Dalla BS et al. Spontaneous entrainment of running cadence to music tempo. Sports medicine. 2015;1(1):1-15.

7. Escher BM, Barbosa BA, Canha OÁ. A influência do espectro de ruído na prevalência de Perda Auditiva Induzida por Ruído em trabalhadores. Braz J Otorhinolaryngol. 2009;1(1):42-7.

8. Samelli AG, Fiorini AC. Ações de proteção para prevenção de perdas auditivas relacionadas ao trabalho In: Boéchat EM, Lemos M, Pedro $C$, Christiane M (orgs). Tratado de audiologia. $2^{\circ}$ edição. São Paulo:Santos; 2015. p 205-10.

9. Lim DJ, Dunn DE. Anatomic correlates of noise induced hearing loss. Otolaryngol Clin North Am. 1979;12(3):493-513.

10. Morest DK, Bohne BA. Noise induced degeneration in the brain and representation of inner and outer hair cells. Hear Res. 1983;9(2):145-51. 
11. Silveira AS, Castro Junior N. Audiometria de tronco encefálico em motoristas de ônibus com perda auditiva induzida pelo ruído. Braz $\mathrm{J}$ Otorhinolaryngol. 2009;75(5):753-9.

12. Nunes CP, Abreu TRMD, Oliveira VC, Abreu RMD. Sintomas auditivos e não auditivos em trabalhadores expostos ao ruído. Rev. baiana saúde pública. 2011;35(3):548-55.

13. Rodrigues TAE. Análise dos comportamentos de condução de sessão e instrução em instrutores de BodyPump® e Indoor Cycling [dissertação]. Castelo Branco (Portugal): Instituto Politécnico Castelo Branco; 2017.

14. Ising $H$, Babisch W, Kruppa B. Noise-induced endocrine effects and cardiovascular risk. Noise and health. 2010;1(4):37-40.

15. Palma A, Mattos UADO, Almeida MND, Oliveira GEMCD. Nível de ruído no ambiente de trabalho do professor de educação física em aulas de ciclismo indoor. Rev Saúde Pública. 2009;43(1):345-51.

16. Caldart AU, Adriano CF, Terruel I, Martins RF, Caldart AU, Mocellin M. Prevalência da perda auditiva induzida pelo ruído em trabalhadores de indústria têxtil. Arq Int Otorrinolaringol. 2006;10(3):192-6.

17. Araújo SA. Perda auditiva induzida pelo ruído em trabalhadores de metalúrgica. Rev Bras Otorrinolaringol. 2002;68(1):47-52.

18. Barros SMDS, Frota S, Atherino CCT, Osterne F. A eficiência das emissões otoacústicas transientes e audiometria tonal na detecção de mudanças temporárias nos limiares auditivos após exposição a níveis elevados de pressão sonora. Rev Bras Otorrinolaringol. 2007;73(5):593-7.

19. Sá LCBD, Lima MAMT, Tomita S, Frota SMMC, Santos GDA, Garcia TR. Avaliação dos limiares de audibilidade das altas frequências em indivíduos entre 18 e 29 anos sem queixas otológicas. Rev Bras Otorrinolaringol. 2007;73(2):215-25.

20. Castro IFC, Conde CA, Paiva APQF, Oliveira LTN, Bernardi APA. Estudo do perfil audiométrico em alta frequência em trabalhadores expostos a ruído. Rev. CEFAC. 2004;6(2):203-8.

21. Ahmed HO, Dennis JH, Badran O, Ismail M, Ballal SG, Ashoor A et al. High frequency (10-18 kHz) hearing thresholds: reliability, and effects of age and occupational noise exposure. Occup. Med. 2001;51(4):245-58.

22. Oliveira PFD, Raposo OFF, Santos ACAD, Santos LAD. Emissões otoacústicas como instrumento de vigilância epidemiológica na saúde do trabalhador. Arq Int. Otorrinolaringol. 2011;15(14):444-9.

23. Heupa AB, Gonçalves CGDO, Coifman H. Effects of impact noise on the hearing of military personnel. Braz J Otorhinolaryngol. 2011;77(6):747-53.

24. Côrtes AIF, Silva SA, Frota SMMC. Estudo das emissões otoacústicas produto de distorção durante a prática esportiva associada à exposição à música. Rev. CEFAC. 2009;11(4):1-8.

25. Marques FP, Costa EA. Exposição ao ruído ocupacional: alterações no exame de emissões otoacústicas. Rev Bras Otorrinolaringol. 2006;72(3):362-6.

26. Lima FGA. Perícia e assistência técnica em audiologia. In: Bevilacqua MC, Martinez MAN, Balen AS, Pupo AC, Reis ACMB, Frota S (orgs). Tratado de Audiologia. $1^{\circ}$ Edição, São Paulo: Ed. Santos; 2011.p.235-42.

27. Ramos N, Domingues CAA, Pillon SL, Sbicigo AF. O uso de emissões otoacústicas como ferramenta auxiliar no diagnóstico de efeitos da exposição ao ruído. Rev Bras Saúde Ocupacional. 2011;36(124):282-7.

28. Martins MVS. Influência de diferentes níveis de pressão Sonora no limiar Auditivo de professores de Ciclismo Indoor [dissertação]. Curitiba (PR): Universidade Federal do Paraná; 2015.

29. Possani LN. Estudo da prevalência e das características do zumbido em trabalhadores expostos ao ruído ocupacional [dissertação]. Porto Alegre (RS): Universidade Federal do Rio Grande do Sul; 2006.

30. Rubak T, Kock S, Koefoed-Nielsen B, Lund $\mathrm{SP}$, Bonde JP, Kolstad HA. The risk of tinnitus following occupacional noise exposure in workers with hearing loss or normal hearing. Int $\mathrm{J}$ Audiol. 2008;47(3):109-14.

31. Steinmetz LG, Zeigelboim BS, Lacerda AB, Morata TC, Marques JM. Características do zumbido em trabalhadores expostos a ruído. Rev Bras Otorrinolaringol. 2009;75(1):7-14.

32. Hyvarinen AS, Pirvola JLU, Ylikoski M, Makitie A, Aarnisalo A, Ylikoski J. Hearing disorder from music: a neglected dysfunction. Acta otolaryngol. 2018;138(1):21-4.

33. Weber SR, Périco E. Zumbido no trabalhador exposto ao ruído. Rev. Soc Bras Fonoaudiol. 2011;16(4):459-65. 
34. Santos CCS, Juchen LS, Rossi AG. Processamento auditivo de militares expostos a ruído ocupacional. Rev. CEFAC. 2008;10(1):92-103.

35. Silva MCBC, Souza MB, Mitre CCL, Ibrahim E. Avaliação do processamento auditivo em operadores de telemarketing. Rev. CEFAC. 2006;8(4):536-42.

36. Xu ZM, Vinck B, De Vel E, Van Cauwenberge P. Mechanisms innoise induced permanent hearing loss: an evoked optoacoustic emission and auditory brainstem response study. J Laryngol Otol.1998;112(12):1154-61.

37. Latjman Z, Borcić V, Markov D, Popović-Kovacić J, Vincelj J, Krpan D. Clinical interpretation of brainstem evoked response audiometry abnormalities in cochlear pathology. Acta Med Croatica.1999;53(1):119-23.

38. Hull RH, Kerschen SR. The influence of cardiovascular health on peripheral and central auditory function in adults: a research review. Am J Audiol. 2010;19:9-16.

39. Kolkhorst FW, Smaldino JJ, Wolf SC, Battani LR, Plakke BL, Huddleston $S$ et al. Influence of fitness on susceptibility of noise induced temporary threshold shift. Med Sci Sports Exerc. 1998:30(2):289-93.

40. Samelli AG, Gomes RF, Chammas TV, Silva BG, Moreira RR, Fiorini AC. The study of attenuation levels and the comfort of earplugs. Noise Health. 2018;20(94):112-9. 


\section{ANNEX 1: Anamnesis Questionnaire}

NAME:

DATE:

AGE: $\quad$ DATE OF BIRTH:

1) Experience time as an indoor cycling teacher?

2) Do you wear personal protective equipment (PPE)?

3) Do you have problems with ear infection or pain?

( )Yes( ) No

4) Do you use any type of medication?

( ) Yes ( ) No

5) Do you have a family history of hearing loss?

( ) Yes ( ) No

6) Are you exposed to noise?

( ) Yes ( ) No

7) Do you have tinnitus in your ear?

( ) Yes ( ) No

8) Do you feel 'dizzy'?

( ) Yes ( ) No

9) Do you have difficulties in following group conversations?

( ) Yes ( ) No

10) Do you feel the need to increase the volume of things: radio or television, when you are in the presence of another person?

( ) Yes ( ) No

Adapted from Campelo, L. M. P; 2007 